\title{
Fracton Contribution to the Electron and Magnon Pairing
}

\author{
Z. BĄK
}

\author{
Institute of Physics, Jan Długosz University, 42-200 Czestochowa, al. AK 13/15, Poland
}

\begin{abstract}
We show that high temperature superconductivity contrary to the Bardeen-Cooper-Schrieffer /BCS/ superconductors exhibits three important energy scales. The highest is associated with the formation of Cooper pairs coupled due to the magnetic fracton excitations, the second (pseudogap) we ascribe to the formation of coherent but not nonsuperfluid condensate. The lowest energy scale (superconducting gap) we associate with the onset of superfluidity of the condensate that is due to the phonon aided fracton hopping.
\end{abstract}

DOI: 10.12693/APhysPolA.126.372

PACS: 84,71.Ba, 74.90.+n, 64.60.al

\section{Introduction}

A century after discovery of superconductivity /SC/ there is no consensus on what kind of thermal excitations contributes to the pair formation. Although the physics of conventional SC with high density of charge carriers is pretty well understood the high temperature $\mathrm{SC} / \mathrm{HTSC} /$ is still an object of controversies. This is because they exhibit many striking features that do not fit the standard BCS phonon-mediated SC [1].

In the paper we address the concept of magnetic fractons based mechanism of SC. In 124 and some other cuprates the scanning $\mathrm{x}$-ray diffraction, with focused synchrotron radiation, shows existence of fractal structures in the proximity of the stripe critical point [2]. The $\mathrm{x}$ ray diffraction of the copper oxides shows, in addition to the main peak, some satellite reflection peaks produced by ordering of oxygen interstitials /O-i/. The satellite peaks are associated with nonuniform (fractal) distribution of the O-i dopants. The probability of distribution of the oxygen dopants is given by the power law $P(x)=x^{\alpha} e^{(-x / x o)}[3]$. The experimental data can be fitted with power law exponent $\alpha=2.6$. This is a direct evidence of the fractal structure. Moreover, the experimental data show that high temperature SC is favoured by the appearance of the fractal structure. This gives us hints for the mechanism behind the SC. Formation of Cooper pairs is not sufficient for the onset of SC, for this we need two additional phenomena to occur. These are: coherence of the pairs ( $\mathrm{BEC}$ transition) and independently superfluidity of the condensate. In the BCS superconductors all the three phenomena arise simultaneously at critical temperature $\mathrm{T}_{C}$, however, there is no need to be so. Temperatures at which these phenomena start their manifestation can be different. As the proof let us point out experimental evidences of preexisting Cooper pairs (above critical temperature) in copper oxides, and the fact that in magnetic systems the magnon Bose-Einstein condensates are not always superfluid. For superfluidity to occur at least the Landau cri-

e-mail: z.bak@ajd.czest.pl terion has to be fulfilled. Indeed, condensation means only that there is the macroscopic phase coherence of condensed quasiparticles. Superfluidity means something more, it occurs only in the case when no new excitations can be created by the condenste due to its motion (no energy dispersion). This means, the condensate which satisfies no Landau criterion behaves as a normal fluid. This means that in some superconductors we can expect three characteristic energy scales (temperatures): i/ the Cooper pair binding energy, (their formation starts at temperature $T^{*}$ ), ii/ temperature of the formation of the coherent state $T^{* *}$ (Bose-Einstein condensation /BEC/ energy pseudogap), iii/ temperature $T_{C}$ at which the condensate becomes superfluid (opening of the SC gap). In our model the superconductivity develops in the three stage process. First there arise preexisting Cooper pairs, coupled due to the magnetic fractons at temperature $\mathrm{T}^{*}$. At lower temperature $T^{* *}$ there occurs the BEC transition and nonsuperfluid condensate is formed. Finally the phonon assisted fracton hopping gives rise to the SC at critical temperature $\mathrm{T}_{C}$.

\section{Fracton based pairing}

The HTC superconductors show quasi-1D, striped hole organization with fractal hole (O-i dopants) distribution within a stripe [3]. With each hole there is associated an extra spin, so not only in elastic but also in the magnetic order we have a fractal component. Therefore in the system we have fracton excitations of both elastic [4] and magnetic nature [5]. Both of them can serve as the pairing agents for the Cooper pairing. Preliminaries of the fractal acoustics (for magnetic excitations the approach is similar) can be found in [6]. The fractional dynamics can be described by a fractional wave equation

$$
{ }_{x} D^{\beta} u=\lambda \cdot{ }_{t} D^{\alpha} u,
$$

where ${ }_{x} D^{\beta}$ and ${ }_{t} D^{\alpha}$ denote the Riemann-Louville fractional diffintegral operators with respect to the space and time variables respectively [6]. The solutions for the amplitude $u$ of the local elastic/magnetic fluctuation is given by $u(x) \propto x^{\alpha} E_{\alpha \beta}(k x)[6]$, where $E_{\alpha \beta}(k x)$ denotes the generalized Mittag-Leffler function which at short distances behaves as the stretched exponential $\exp \left(k x^{\alpha}\right)$. In spite of theoretical divisions regarding the role of mag- 
netic excitations in cuprate, it seems that there are more experimental data speaking in favour of magnetic mechanism of pairing. Moreover, numerical simulations show that in cuprates the dominant contribution to the pairing interaction is associated with the spectral region characteristic of the spin fluctuations [7]. As the Cooper pairs are singlets they should carry two magnetic fractons with opposite magnetic moments. This concept resembles biphonon Cooper aggregates discussed in [8]. At higher temperatures the Cooper pairs are in normal state and are localized within stripes. When such Cooper pairs condense there arises coherence not only in the electron/hole system but also in the magnetic excitations. As the argument for such a scenario let us recall experimental data. Neutron measurements of magnetic excitation spectra in YBCO show apparent weakness of antiferromagnetic fluctuations in the optimally doped YBCO compounds [9]. The integrated intensity of these excitations constitutes only a few percent of the spectral weight of spin waves in magnetically ordered 123 systems. We interpret this as partial condensation of magnetic excitations that reduces population of uncorrelated excitations visible to neutrons. When the temperature is lowered there arises a BEC phase transition at temperature $\mathrm{T}^{* *}$. It is evident that the condensate with pseudogap $\Delta^{* *}$ is not superfluid. Localization of the fractons within the stripe structure results in pinning of the condensate. However, at lower temperatures the tails of the fracton solutions overlapping, enhanced by phonons, can create an interstripe coupling, which eventually enables superconductivity [10]. This means that two different interactions of magnetic and elastic nature contribute to the formation of SC. Currently there are two alternative explanations of the pseudogap. This is a precursor of the superconducting gap without long range coherence, or this is a normal state which is independent from the superconducting gap. In our model we argue for the third explanation, the pseudogap is associated with a BEC transition to a nonsuperfluid condensate. The superconductivity arises later when condensate decouples from the stripe structure.

\section{Coexistence of two order parameters}

The most often used theoretical formalism for the description of nonuniform superfluid condensates is the Gross-Pitaevski formalism. The local complex order parameter of $\Psi(r, t)$ can be found from the Gross-Pitaevski equation. Writing down the free energy functional $F$ in terms of $\Psi(r, t)=\Psi(r) e^{i \omega t}$.

$$
F-\mu \Psi=\int \mathrm{d}^{3} r\left(\frac{|\nabla \Psi|^{2}}{2 m}+\left[\omega_{L}(r)-E_{D}\right]|\Psi|^{2}\right),
$$

the Gross-Pitaevski formalism reduces to the landauGinzburg approach. As it was shown [10] in fractal systems the gradient term $|\nabla \Psi|^{2}$ in Eq. (2) should be replaced by the fractional gradient term $\left.\sum_{i}\right|_{x_{i}} D^{\beta} \Psi_{x_{i}} D^{\beta} \Psi \mid$. Under the assumptions above the free energy of the ordered fractal system, this results in the free energy of the system which takes the form [11]

$$
\begin{aligned}
F & =F_{0}+\int\left[\left.g_{\lambda} \sum_{i}\right|_{x_{i}} D^{\beta} \Psi_{x_{i}} D^{\beta} \Psi \mid\right. \\
& \left.+a|\Psi|^{2}+\frac{b_{\lambda i}}{2}|\Psi|^{4}\right] \mathrm{d} \boldsymbol{r} .
\end{aligned}
$$

As we assume the existence of two order parameters associated with the two phase transitions at temperatures $T^{*}$ and $T_{C}$, we should use a generalized expansion of the Landau-Ginzburg free energy functional $F$ in terms of two order parameters $\Psi_{1}$ and $\Psi_{2}$ associated with these transitions. The simplest expansion of the free energy takes the form of

$$
\begin{aligned}
F & =\frac{1}{2} \int \mathrm{d}^{3} r\left[\frac{1}{\chi_{1}}\left|\Psi_{1}\right|^{2}+\frac{1}{\chi_{2}}\left|\Psi_{2}\right|^{2}\right. \\
& \left.+g_{2}^{1}\left|\Psi_{1}\right|^{2}\left|\Psi_{2}\right|^{2}+\frac{1}{2} g_{1}\left|\Psi_{1}\right|^{4}+\frac{1}{2} g_{2}\left|\Psi_{2}\right|^{4}\right] .
\end{aligned}
$$

The minimization of the free energy allows to determine the equilibrium conditions of coupled parameters of order. The variety of solutions can be found in [11]. The most important conclusion from these solutions is that under some combination of the parameters $g_{i}$ and $g_{i}^{j}$ in Eq. (4) the nonzero values of both order parameters $\Psi_{1}$ and $\Psi_{2}$ can coexist at temperatures higher than those predicted by a model with no coupling between them. This means that $\mathrm{SC}$ onset temperature $T_{C}$ can be enhanced by the coupling with the other condensate.

In summary, in our study we give arguments that $\mathrm{SC}$ in HTSC can be interpreted in terms of three stage process. First there arise Cooper pairs which are bound via magnetic fractons. At lower temperature they condense into a nonsuperfluid condensate. Finally, at the third stage there arises superfluidity (and superconductivity) in the electron/fracton condensate.

\section{References}

[1] S. Kumar, S.N. Kaul, J.R. Fernandez, L.F. Barquin, J. Appl. Phys. 105, 078901 (2009).

[2] S. Kambe, Y. Ichimaru, E. Sato, C. Yoshida, O. Ishii, Bull. Yamaganta Univ. (Eng) 27, 13 (2002).

[3] N. Poccia, A. Ricci, A. Bianconi, J. Supercond. Nov. Magn. 24, 1195 (2011).

[4] Z. Bak, Materials Science-Poland 26, 913 (2008).

[5] Z. Bak, Acta Phys. Pol. A 113, 541 (2008).

[6] Z. Bak, in Classification and Application of Fractals, chapter 12, Ed. William L. Hagen, ISBN 978-1-61209967-5, Nova Science Publishers Inc., NY 2011.

[7] T.A. Maier, D. Poiblanc, D.J. Scalapino, Phys. Rev. Lett. 100, 237001(2008).

[8] Z. Bak, Czech. J. Phys. 54, 461 (2004).

[9] M.Le Tacon, G. Ghiringhelli, J. Chaloupka, M. Moretti Sala, V. Hinkov, M.W. Haverkort, M. Minola, M. Bakr, K.J. Zhou, S. BlancoCanosa, C. Monney, Y.T. Song, G.L. Sun, C.T. Lin, G.M. De Luca, M. Salluzzo, G. Khaliullin, T. Schmitt, L. Braicovich, B. Keimer Nature Physics 7, 725 (2011).

[10] A.V. Milovanov, J.J. Rasmussen, Phys. Rev. B 66, 134505 (2002).

[11] Z. Bak, Phase Transitions 80, 79 (2007). 